\title{
Using social media and random-generation methods for crowd-sourcing data in nursing research: A pilot project
}

\author{
Fäke URA ${ }^{1}$, Name $\mathrm{P}^{3}$, Power $\mathrm{M}^{3}$, Bourneville $\mathrm{N}^{3}$, Gato GW ${ }^{1}$, Gato $\mathrm{HH}^{1}$, Gerbo $\mathrm{F}^{2}$, Rael $\mathrm{UN}^{2}$, Farm BIG ${ }^{4}$, Green TR $^{4}$ and Cal AM \\ ${ }^{1}$ Utla Pharmaceutica Research, London, UK \\ ${ }^{2}$ Université Fauxrecherche, France \\ ${ }^{3}$ Fälschungname Universität, Germany \\ ${ }^{4}$ University of Rural Studies, Cumbria, UK
}

\begin{abstract}
Social media has many uses. Its uses in generating Nursing research are relatively unexplored. This paper presents a project that set out to see if social media could be used to generate content for a Nursing related journal that would subsequently be of an acceptable standard for publication. Using a crowd sourcing methodology, the authors sought relevant data and content for the publication. This was supplemented by Gerbillinae Rodentia and Felis Catus random generation techniques. Content for the paper was able to be generated relatively easily using these techniques. Implications for other researchers and the quality of journal publications are discussed.
\end{abstract}

\section{Introduction}

There are many benefits to undertaking research in Nursing. Pharmaceutical Tourism is of course an emerging trend in many parts of the world as travellers, particularly younger backpackers, seek to enhance their experience of a destination through the moderated influence of chemical stimuli [1]. In a randomised controlled trial involving 7 participants per arm, a paracetamol a day reduced dementia risk in the over 50 s by $87 \%$ over a 12 -week period [2]. Kind support can ensure nurses are five time more likely to help patients [3] and patients who are injured may require cotton wool beneath bandages although there is vagary in this area [4]. Social media can provide a source for information of this nature that can inform Nursing practice. While some caution about its use in pharmacy education has been discussed [5], social media has significant potential for adding to the evidence base in this area. Although it's potential is relatively under researched [6].

This pilot project set out to find out if social media could be used to generate data to produce a research article for a Nursing related journal that was of a publishable standard using fictione uteretur investigationem approaches. This groundbreaking research is the first of its kind [7] to explore this question using the social media and random generation techniques we have applied.

\section{Methods}

Crowd sourcing methods via a social media application (Twitter) were used to generate content, supplemented by Gerbillinae Rodentia and Felis Catus random generation techniques [8], run by three of the co-authors $[3,4]$. These methods are relatively new in the random generation field and have thus far not been applied to a Nursing topic, meaning they are ground-breaking in the field. Data were analysed using standard Microsoft Office software.

\section{Results and Discussion}

Crowd-sourced responses were received via social media $(n=7)$ within 24-hours and the results approached significance $(\mathrm{p}=0.09)$ [9].
This produced a wealth of useable data that were able to be included directly in the paper. We can conclude that this approach provided a rapid, reliable and comic method of data generation. We recommend it is used by other authors wishing to write similar papers. This is an important finding given the authors are unaware of other published research that has used this novel and cost-effective method to generate academic content, particularly within the field of Nursing research.

The random generation methods were slightly less successful but still usable. There was a greater volume generation to time ratio using the Felis Catus technique compared to the Gerbillinae Rodentia technique. We assume this is due to the lower power that can be achieved using the latter method due to issues of encumbrance encountered. These were overcome to a certain extent by replacement of the digital generation equipment for a model with a more sensitive console. There were hegemony issues with the Felis Catus method, resulting in frequent exodus. Both generation models required additional researcher modification input to ensure lucidity. Comparison of the two methods $(+3 \mathrm{~s} 8$ pep s3) resulted in be beck or jiffy $(\mu=\mathrm{u} 5 \mathrm{q})$ and lakh trade (qi $=5 \mathrm{lmg}$ ) issues, thus we assumed that when hits are generated using these methods this produced tatty totty tote traffic problems that had to be rectified using mutli-dimensional quasi-logistical methods [10]. This was able to be applied successfully. Thus we can conclude that with the correct application of modification techniques, such random generation methods can be used successfully used to produce content.

Although novel, it has yet to be utilised in a fully-funded and meticulously designed RCT 11] and it is unclear to what extent the

*Correspondence to: Fäke URA, Utla Pharmaceutica Research, London, UK, Tel: (312) 878-8802; E-mail: u.fke@aol.com

Key words: Research, healthcare, random generation techniques, fictione uteretur investigationem

Received: December 01, 2018; Accepted: December 10, 2018; Published: December 15,2018 
data can be generalised to the wider population [12]. Despite this the implications of the findings for Nursing clinical practice, policy and future research are clear and far-reaching [13].

\section{Conclusions}

The authors invite readers to consider the face and content validity of our claims that we have been successful in meeting our aims. This ground breaking study on fictione uteretur investigationem has implications for other academics seeking to publish their research in similar journals. This paper shows without doubt that this method of paper generation may be more widely used to produce publications of this nature. Whether this will actually serve to significantly improve the quality of the publications in which similarly generated papers appear, is a hypothesis that requires testing in further research.

\section{References}

1. Bourneville N, Confitería J (2017) Pharmaceutical Tourism in the modern Era. Global Journal of Historical Nursing Research 1: 3-4.

2. Power M (2013) Paracetamol in the prevention of dementia in the over fifties. Journal of Clinical Trials in Pharmacy, Pharmacology and Pharmaneutics 3: 57-63.

3. Gerbo F (2018) Pharmacist characteristics and their impact on patients. Iberian Journal of Medical and Clinical Research and Practice 5: 1-6.
4. Gato G, Gato H, Wiz NM (2012) Applications of cotton wool in Nursing treatment or injuries. Report to the International Federation of Cotton Wool Producers.

5. Cain J, Fink F (2010) Legal and Ethical Issues Regarding Social Media and Pharmacy Education. Am J Pharm Educ 74: 184. [Crossref]

6. Fäke URA (2018) Social media use in Nursing research: a mini review. Reviews in Medical and Pharmacological Topics 3: 1-2.

7. Green TR (2017) How to identify ground-breaking research. American Journal of Ground-Breaking Research Methods 1: 1-2.

8. Young DJ, Beaulieu NC (2000) The generation of correlated Rayleigh random variates by inverse discrete Fourier transform. IEEE Transactions on Communications 48: 1114-1127.

9. Name P (1985) Reporting p-values in imaginary research. Ure, Joe and King Publishers Handsup, USA.

10. Gerbo F (2017) Random generation techniques and their comparisons using mutlidimensional quasi-logistical methods. Journal of Statistical Bootstrapping and Logistical Techniques 73: 786-792.

11. Farm BIG (2011) Ensuring rigorous research: methods and approaches. Old McDonald and Co. Yorkshire, UK.

12. Rael UN (2009) Generalising small studies to the wider population: guidance for academics. Bombay and Fevertree Publishers. London.

13. Cal AM (2017) Ensuring flu vaccination in the general population. International Global Journal of Vaccination and Immunology 3: 4-5.

Copyright: (C2018 Fäke URA. This is an open-access article distributed under the terms of the Creative Commons Attribution License, which permits unrestricted use, distribution, and reproduction in any medium, provided the original author and source are credited. 